\title{
GWAS-MAP: the platform for analysis of results of genome- wide association studies
}

\author{
Tatiana Shashkova \\ Laboratory of Theoretical and Applied \\ Functional Genomics \\ Novosibirsk State University \\ Novosibirsk, Russia \\ t.shashkova@nsu.ru \\ Sodbo Sharapov \\ Laboratory of Theoretical and Applied \\ Functional Genomics \\ Novosibirsk State University \\ Novosibirsk, Russia
}

\author{
Denis Gorev \\ Laboratory of Theoretical and \\ Applied Functional Genomics \\ Novosibirsk State University \\ Novosibirsk, Russia \\ Yakov Tsepilov \\ Laboratory of Theoretical and \\ Applied Functional Genomics \\ Novosibirsk State University \\ Novosibirsk, Russia \\ Yurii Aulchenko \\ Laboratory of Theoretical and \\ Applied Functional Genomics \\ Novosibirsk State University \\ Novosibirsk, Russia
}

\author{
Eugene Pakhomov \\ Laboratory of Theoretical and Applied \\ Functional Genomics \\ Novosibirsk State University \\ Novosibirsk, Russia \\ Lennart Karssen \\ PolyKnomics \\ 's-Hertogenbosch, Netherlands
}

\begin{abstract}
We present the database and the highperformance GWAS-MAP platform for aggregating, storing, processing and visualizing big data, namely the results of genome-wide and regional association studies (GWAS and RWAS). At the moment, we have compiled a database of human GWAS and RWAS, which contains more than 70 billion associations between genomic polymorphisms and different diseases, quantitative and "omics" traits. The platform and the database can be used in studies of the etiology of human diseases, in the development of predictive risk models, as well as in the search for candidate biomarkers, and candidate therapeutic targets.
\end{abstract}

Keywords - GWAS-MAP, database, genome-wide association study, summary statistics, GWAS, regional-wide association study, RWAS, quantitative genetics

\section{Introduction}

The genome-wide association study (GWAS) is one of the main approaches for identifying the relationships between allelic variants of the genome and human complex traits. One of the most important advantages of GWAS is the agnostic nature of the method. The GWAS methodology has gained great popularity over the past decade [1]. Since 2007, the number of GWAS has been growing exponentially; At present, hundreds of original genome-wide association studies are published each year.

The results of GWAS are published in the form of summary statistics - for each single nucleotide polymorphism (SNP) information is provided on the frequencies of alleles and characteristics of the effect of the allele on the phenotype. For the analysis of human genomes, a GWAS assumes a study of associations between the character of interest and tens of millions of SNPs. In this regard, the results of GWAS (RGWAS) form big data.

R-GWAS can be used to solve many problems - from research in the field of fundamental biology and genetics to the search for biomarkers and therapeutic effects.

While the number of R-GWAS collected by the scientific community and their analysis methods is increasing, the use of this data is limited by the lack of generally accepted standards. In particular, researchers are faced with the following problems. Firstly, this is a big amount of data that requires the development of specialized infrastructure. Secondly, in addition to storing primary data, it is necessary to ensure their pre-processing. Thirdly, the same type of data is generated in different laboratories using different protocols, which requires an integration procedure to ensure universal storage. Finally, it is necessary to develop user interfaces that would allow non-bioinformaticians access and analyze the data.

To help addressing these issues, we developed the GWASMAP platform for storing, processing, and analysis of RGWAS. The system provides an opportunity to carry out research that will contribute to the search for new candidate biomarkers and therapeutic approaches.

\section{Results}

The GWAS-MAP consists of two data processing modules (integration and analysis of GWAS/RWAS results), an interface to the database (DB) and a web-interface (Fig. 1). Integration involves converting R-GWAS, collected from different sources, into a universal format. After integration, we perform QC and, if the R-GWAS passes the tests, we keep it in the database.

The DB consists of two components, each controlled by its own DB management system (DBMS). The first component is managed by the Clickhouse DBMS, which provides us with rapid-access storage of RGWAS accessible via powerful and flexible interface. Each record is a vector of parameters characterising an association between a SNP and a trait in a GWAS. The second component is managed by the PostgreSQL DBMS and contains information about the study design, results of analyses and other supporting information. To ensure convenient usage of GWAS data, the system also contains key information about each study, for example, date of publication, reference, and details of the experiment.

Currently, the GWAS DB contains in total data from 7,245 genome-wide and 1,25 millions regional (e.g. cis-eQTL) association scans describing 70 billions of genotypephenotype associations. Tirty-six percents of associations are 
related to complex traits, $12 \%$ are related to transcriptomics, $4 \%$ - to metabolomics, $47 \%$ - proteomics and $1 \%$ - glycomics. To ensure convenient usage of GWAS data, the system also contains key information about each study, for example, date of publication, reference, and details of the experiment.

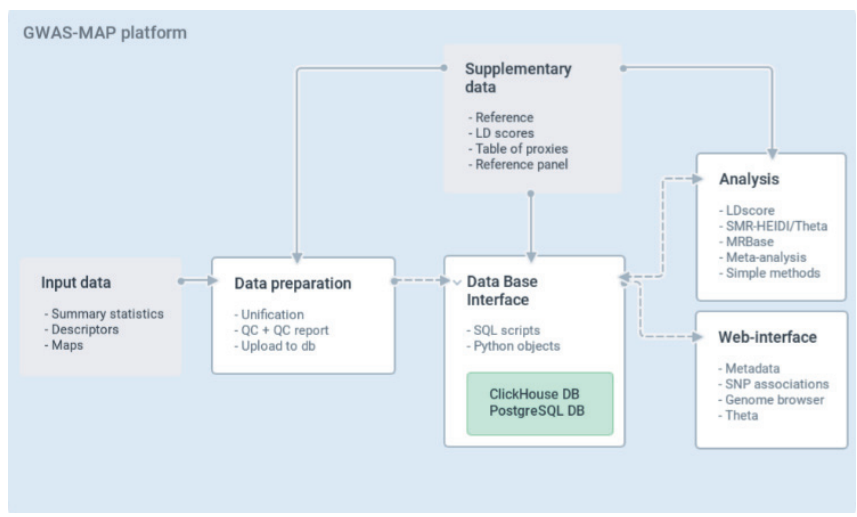

Fig. 1. GWAS-MAP platform program architecture

The system incorporates widely used methods of RGWAS analysis. These methods are focused on the identification of functional variants, genes, molecules, and traits that may be potential targets of (therapeutic) manipulation. In particular, we implemented methods of

- Linkage Disequilibrium score regression - a method for assessing the SNP-heritability of a trait and calculating genetic correlations between two traits [2].

- Mendelian randomization methods - a set of tests that allow hypothesising a causal relationship between a pair of traits [3].

- Summary-level mendelian randomization and heterogeneity in dependent instruments (SMRHEIDI) which helps determining whether two different traits are controlled by the same functional variant in the locus or are controlled by two different functional variants in linkage disequilibrium. When used with gene expression GWAS, this can permit the establishment of the causal gene in a locus, which is not readily attained using a trait GWAS alone [4].

- Dimitrieva-Georges Theta (DGT) - is an alternative to HEIDI. This method uses weighted correlation analysis to evaluate the similarity of association profiles based on summary statistics only. The method may be more robust than SMR/HEIDI because it does not rely on availability and precise knowledge of the structure of linkage disequilibrium $[5]$.

We also implemented meta-analysis tools that allow combining results of GWAS performed for the same trait. This module has additional QC procedure, to check that studies could be included in analysis together [6].

Finally, we developed a web-service "PheLiGe" (https://phelige.com), in order for external users to have access to the database. PheLiGe allows not only for scanning the phenome for effects of a genetic variant, but also to assess whether co-association of a variant with multiple traits may occur due to pleiotropy or the consequence of linkage. In more detail, in the "GWAS/cisQTL" tab, a user can search for specific GWAS and RWAS on multiple criteria, access metadata and generate interactive Manhattan plots. In the "Associations" tab the user can search for associations observed for a SNP of interest directly or via a proxy variant in linkage disequilibrium. The user can download the results and also graphically explore regional pattern of association around the selected SNP. Next, the user can select a "primary" and "secondary" regional association, that will be passed to the "Analysis" tab. In this tab, regional patterns of association are compared using the Dmitrieva-Georges weighted correlation method.

\section{Conclusion}

In this work, we introduced the GWAS-MAP platform, intended for storage and processing of GWAS and RWAS results. Currently, the database system contains data on more than 70 billion genotype-phenotype associations. The system interface provides a universal way of working with data, which allows quickly embed new analysis methods in the system. Users can work with the system through command line utilities, with the help of which they have the ability to download data to the system, as well as run analyzes and get their results. Approbation of GWAS-MAP platform is presented in an article on varicose veins study [7].

\section{ACKNOWLEDGMENT}

This work was funded by the TOP100 program of the Novosibirsk State University and by PolyKnomics BV.

\section{REFERENCES}

[1] Visscher P. M. et al. "10 years of GWAS discovery: biology, function, and translation" The American Journal of Human Genetics, 2017, pp. $5-22$.

[2] Bulik-Sullivan B. K. et al. "LD Score regression distinguishes confounding from polygenicity in genome-wide association studies" Nature genetics, 2015, pp. 291.

[3] Hemani G. et al. "MR-Base: a platform for systematic causal inference across the phenome using billions of genetic associations" bioRxiv, 2016, pp. 078972.

[4] Zhu Z. et al. "Integration of summary data from GWAS and eQTL studies predicts complex trait gene targets" Nature genetics, 2016, pp. 481.

[5] Momozawa Y. et al. "IBD risk loci are enriched in multigenic regulatory modules encompassing putative causative genes" Nature communications, 2018, pp.2427.

[6] Winkler TW et al. "Quality control and conduct of genome-wide association meta-analyses" Nature Protocols, 2014, pp. 1192-1212

[7] Shadrina AS., et al. "Varicose veins of lower extremities: Insights from the first large-scale genetic study" PLoS genetics, 2019, pp. e1008110. 